\title{
Review
}

\section{Improvement of Polypropylene (PP) Dyeing by Modification Methods}

\author{
S.M. Gawish*, A.M. Ramadan and S. Mosleh \\ National Research Centre, Textile Division, Cairo, Egypt.
}

$\mathbf{P}$ OLYPROPYLENE fabrics are important for the woven or nonwovens market. PP fabrics have wide applications in our life. PP are difficult to be dyed by conventional dyeing methods, since they have no reactive functional groups in their chemical structure. For the above reasons. PP fabrics are modified by different methods to improve their dyeing property using the following:

I. Blending PP chips with other polymers with different ratios of PTT, PE, PET, EVA, PS, PA during melt extrusion.

II. Dyeing PP using disperse dyes in superfacial carbon dioxide.

III. Mass coloration of PP by melt extrusion with organic, inorganic pigments or metallic additives.

IV. Modification of PP by radio-grafting technique.

V. Dyeing PP by other means.

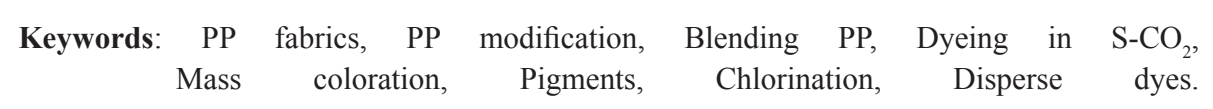

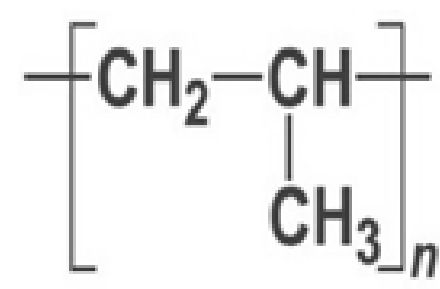

Fig. 1. Chemical structure of Polypropylene.

\section{Introduction}

Polypropylene bears no reactive functional groups in its chemical structure and has no affinity for normal dyestuffs. PP fibers are dyed in pale shades with a limited number of disperse dyes Polypropylene is a major textile for the nonwovens industry and provides $10 \%$ of the market products. PP has high mechanical strength and resistance to chemicals or abrasion. However, its high hydrophobic character combined with the buildup of static charges and resistance to finishing are undesirable properties. Dyed PP fabrics are important and have a wide range of applications such as nonwoven dark color for vehicles lining, fabrics such as cars, trains, airplanes, blue fabrics for nonwoven disposable uniforms, antibacterial sheets and wound healing dressings in medical hospitals in addition to soldiers uniforms. Also, PP fibers are dyed with disperse dyes for use in carpets, rugs, upholstery, swim and sport wear fabrics. Review of the different methods of dyeing polypropylene includes the following:

I . Blending PP chips with other polymers

II. Dyeing PP using superfacial carbon dioxide.

III. Mass coloration with metallic additives and pigments

IV. Modification PP fabrics with selected monomers by radio-grafting techniques

V. Dyeing PP by other means

Blending PP chips with other polymers

PP based polymer fibers containing various quantity $(5-20 \%)$ of poly trimethylene terphthalate (PTT) were melt spun to modify PP structure[1]. The structure behavior change of blended fibers was analyzed by different methods. The poly blend fibers had increased dyeability with increase in PTT content along with excellent color and light fastness properties.

${ }^{*}$ Corresponding author e-mail: smgawish@yahoo.com 


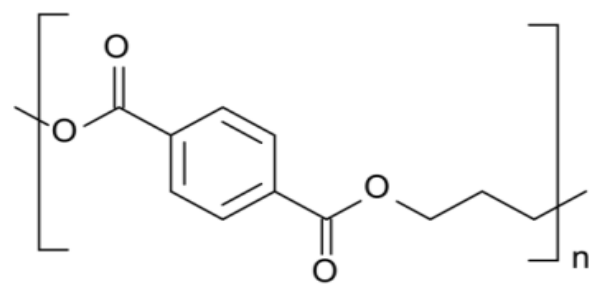

Fig. 2. Chemical structure of poly trimethylene terphthalate (PTT).

Disperse Blue C.I. 79 was used to dye 100 $\%$ isotactic polypropylene (PP) fibers, which are widely applied in the industry. Linear low density $100 \%$ polyethylene (LLDPE) polymer fibers and ternary polymer blends were obtained from four different weight ratios of these polymer fibers with an elastomer of ethylene-vinyl acetate (EVA) in the absence and presence of microwave [2]. Addition of up to $15 \%$ EVA in the ternary polymer blends yields good color and mechanical properties for both dyeing methods In presence of microwave for dyeing, it gave a saving of almost
$90 \%$ of time required and a high amount of energy.

A disperse dyeable PP fiber has been prepared by admixing chips of polystyrene to PP polymer prior to extrusion[3]. Polystyrene has been synthesized at suitable and narrow molecular weight distribution. The dyeability, mechanical and other properties, of pure oriented PP or the blended fibers with different amount of polystyrene were studied. The structure of the blend containing 4 and $6 \%$ polystyrene was studied to explain the interesting dyeing behavior of the blended fibers.

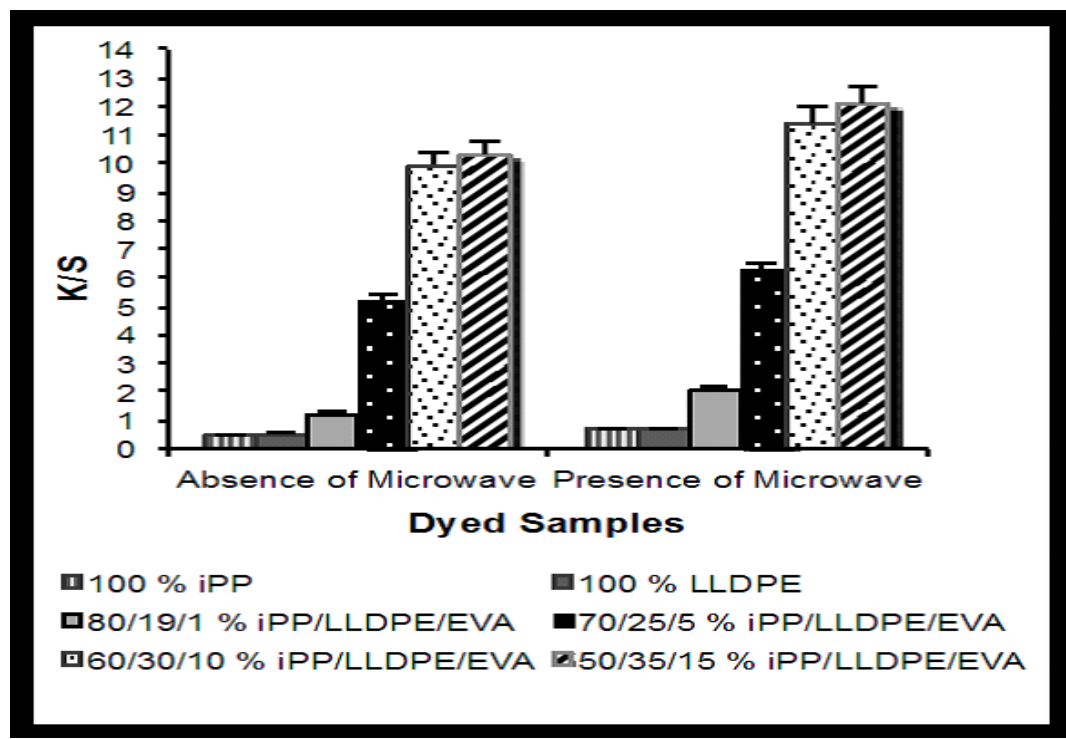

Fig. 3. K/S values of dyed samples at maximum absorption wavelengths $(600 \mathrm{~nm})$.

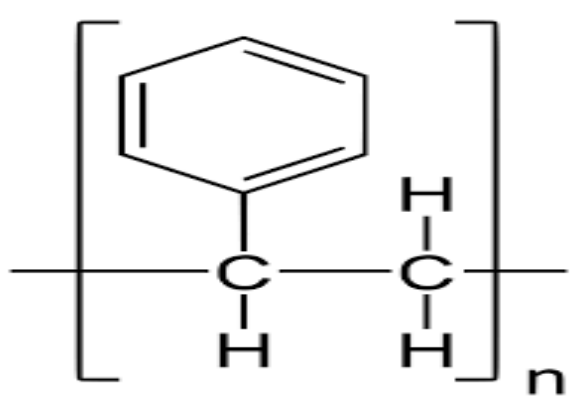

Fig. 4. Chemical structure of polystyrene. 
TABLE 1. Characteristics of disperse dyes

\begin{tabular}{cccc}
\hline Commercial name & Generic name & Chemical structure & Activation energy level \\
\hline Terasil Blue BGE & C.I.Disperse Blue60 & Anthraquinone & Medium \\
Terasil Yellow 4G Terasil & C.I.Disperse Yellow & Monoazo & Medium \\
Terasil Red R & C.I.Disperse Red324 & Monoazo & Medium \\
\hline
\end{tabular}

TABLE 2. Influence of PS content on the properties of blended fibers.

\begin{tabular}{|c|c|c|c|c|}
\hline PP/ PS blends & $\begin{array}{c}\text { Tensile strength at } \\
\text { break (CN/dtex) }\end{array}$ & $\begin{array}{c}\text { Elongation at break } \\
(\%)\end{array}$ & Crystallinity (\%) & $\begin{array}{c}\text { Orientation } \\
\qquad(\%)\end{array}$ \\
\hline Pure PP & 3 & 19.6 & 58.8 & 90.1 \\
\hline PP/PS (98/2) & 2.4 & 22.9 & 50.4 & 88.2 \\
\hline PP/PS (96/4) & 2.2 & 24.5 & 44.3 & 87 \\
\hline PP/PS (94/6) & 2.2 & 25.8 & 40.2 & 86.1 \\
\hline PP/PS (92/8) & 2.1 & 26.5 & 38.1 & 85.6 \\
\hline
\end{tabular}

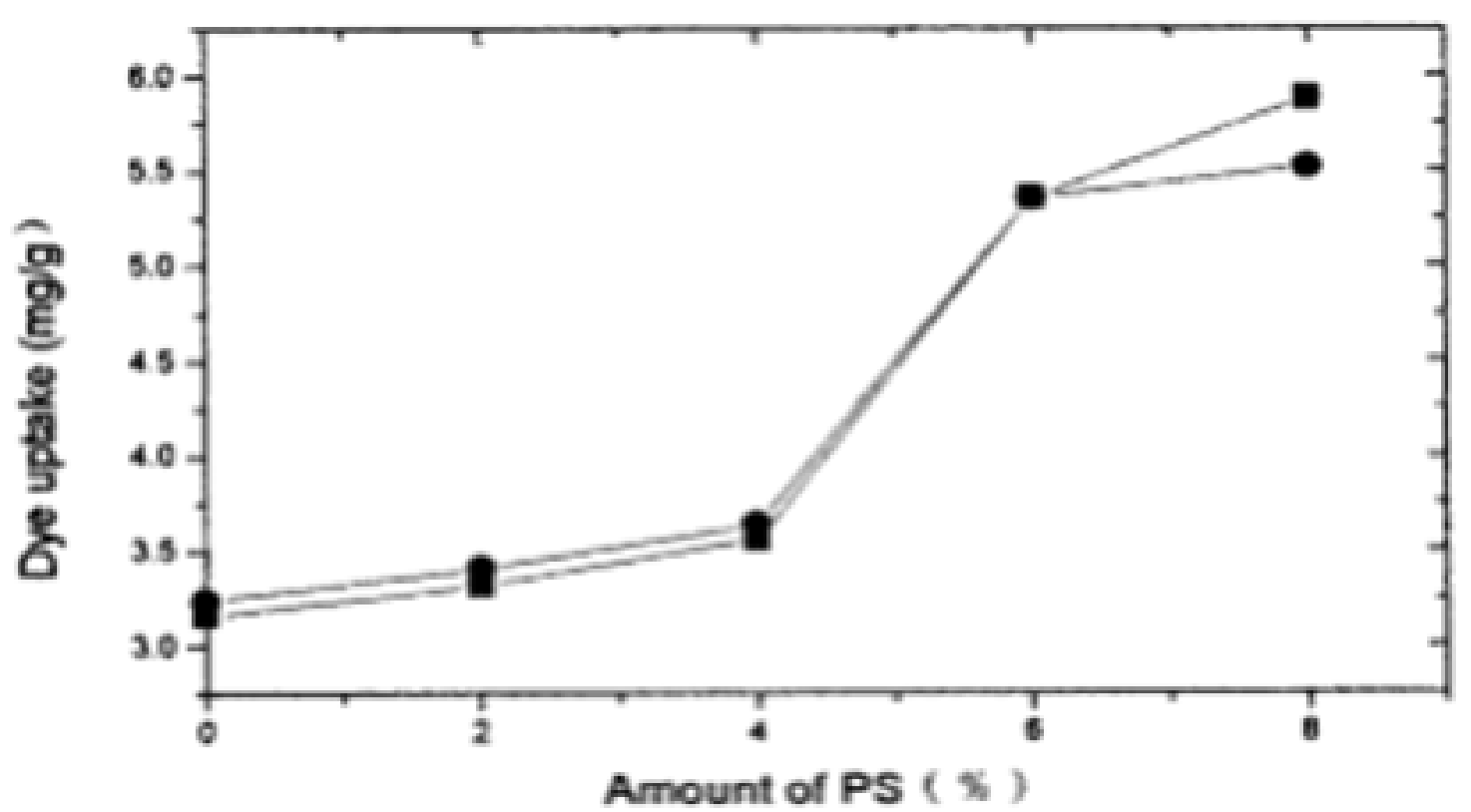

Fig. 5. Influence of amount of PS on dye uptake: (ø) C.I. dispersed red 60; (•) C.I. dispersed yellow 23. 
Dyeable modified polypropylene (PP) fabric with disperse dye was developed [4]. Modified PET chips and PP for compounding processing with a compatibilizer were used. Engineering method was applied to analyze the optimum spinning process parameters. Color and light fastness were evaluated for PP modified and disperse dyed fabric. The optimum processing parameters to develop dyeable PP fiber were studied. The modified PP fabric was made into fibers by melt spinning. The processing parameters for the quality characteristics of tensile strength and elongation were studied and the optimum fully drawn yarn was done. The fibers tensile strength and elongation at optimum parameter design were evaluated. The fibers derived from the optimum parameters are woven into fabric for the disperse dyeing test and the washing fastness was leveled to 3-4. The developed modified PP fabric had good dye-uptake and fastness properties.

Polypropylene chips were modified by melt blending with PET for improvement the dyeability of the modified fibers. Five blends of PP/ PET/ compatilizer were prepared and then spun into fibers. Three disperse dyes were used to dye the modified fibers at $130^{\circ} \mathrm{C}$. The dyeing performance of the blended fibers and the other properties were determined by different methods [5].

TABLE 3. Comparison between optimization of dyeable PP fiber and pure PP fiber.

\begin{tabular}{ccc}
\hline Sample & Tensile strength $(\mathbf{g} / \mathbf{d})$ & Elongation $(\%)$ \\
\hline Pure PP fabric & 3.36 & 36.66 \\
Optimization dyeable PP fabric & 3.36 & 38.65 \\
\hline
\end{tabular}

TABLE 4. Washing and light fastness of various fabrics.

\begin{tabular}{ccc}
\hline Samples & Washing fastness & Light fastness \\
\hline Pure PP fabric & $1-2$ & $1-2$ \\
Optimization dyeable PP fabric & $3-4$ & $2-3$ \\
\hline
\end{tabular}

PP: Polypropylene

TABLE 5. Characteristics of disperse dyes.

\begin{tabular}{lcl}
\hline Generic name & Chemical structure & Activation energy level \\
\hline C.I.DisperseBlue60 & Anthraquinone & Medium \\
C.I.DisperseYellow & Monoazo & Medium \\
C.I.DisperseRed324 & Monoazo & Medium \\
\hline
\end{tabular}

The dyeing of unmodified polypropylene (PP) fibres by the exhaustion method is characterized by a low PP affinity to dyes and low colouring parameters in the dyed fibers. The preparation of polypropylene/ polyester (PP/PET) blend of fibers is interesting from dyeability view of these fibers in dye bath. Polyesters addition to PP matrix is

Egypt. J. Chem. 62, No.1 (2019) able to fix disperse dyes and increase the dye uptake from a dyeing bath.

The influence of two polyesters (polyethylene terephthalate PET and polybutylene terephthalate PBT) for the dyeing kinetics of PP/PET blend fibres with disperse dye C.I. Disperse Blue 56 and the dyeing parameters were studied [6]. 


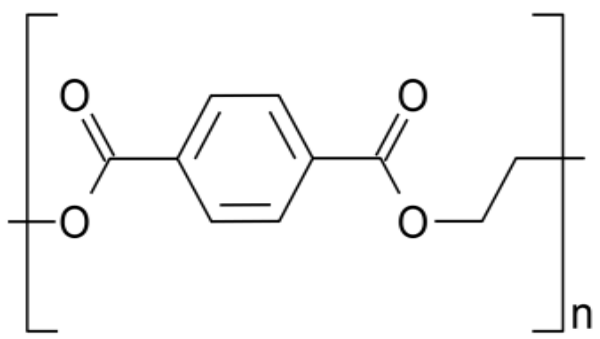

Polyethylene terephthalate(PET)

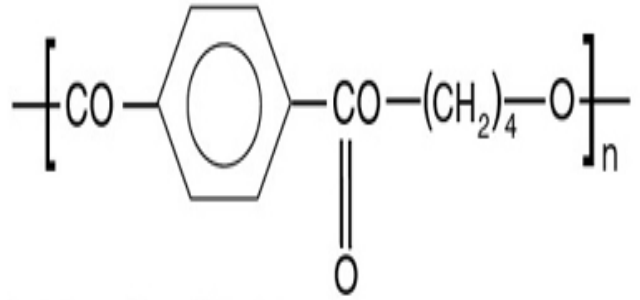

Polybutylene terephthalate (PBT).

Fig. 6. Chemical structures of polyethylene terephthalate (PET) and polybutylene terephthalate.

TABLE 6. Characteristics of blend PP/PET and PP/PBT.

\begin{tabular}{cccc}
\hline \multicolumn{2}{c}{ Sample } & \multicolumn{2}{c}{ Composition of fibers } \\
\hline No. & PP (wt.\%) & PET or PBT(wt.\%) & Compatibilizer(wt.\%) \\
\hline 1 & 90.5 & 8 & 1.5 (OLPET) \\
2 & 90.5 & 8 & 1.5 (EDSA) \\
3 & 80.5 & $8(\mathrm{PBT})$ & 1.5 (EDSA) \\
\hline
\end{tabular}

Compatibilizer: (EDSA) ethylenedistearamide, OLPET oligomeric polyester.

The preparation of PP/ PET blend fibers is important for dyeability. The incorporation of PET to PP [6] has resulted in an appreciable increase in disperse dye-uptake. The chemical nature of the additive and the structural changes of the fibers due to blending determine the dyeing ability. The authors reported that blending with PET decreases PP crystallinity and this is reflected in an increase in disperse dyeing.

Modified PP fibers are dyed with both acid and basic dye using conventional dyeing methods. Various polymeric additives including polyamides, polyamino triazoles, polyamino acrylates have been applied to produce very good dyeable compositions of PP with disperse dyes [7-16]. PP has an important role such as high melting temperature, low density, high chemical resistance to be modified with addition of other polymers or mineral materials and to be grafted with active functional groups [17].

Polypropylene fibers were dyed with disperse dyes and have been prepared by melt blending with polyethylene-co-vinyl acetate (EVA) chips prior to extrusion [18]. Mechanical properties of the modified fibers were investigated. Fibres with various amounts of EVA were dyed with disperse dyes and the dyeing of these fibres were studied. The influence of different proportions of vinyl acetate in the EVA formulation was taken into consideration.

Improvement of disperse dyeablility and imparting cationic dyeablility to difficultly dyeable PP was done by a melt blending technique [19]. Isotactic PP chips were blended with chips of polybutylene terephthalate (PBT), cationic dyeable polyethylene terephthalate (CDPET) and polystyrene (PS), individually. The resulting binary fibers blends were spun and drawn into fibers at different draw ratio. The compatibility of blends was examined by different analysis methods. The blended fibres were disperse dyeable by the conventional method of at high temperature and pressure. PP/CDPET blend also gave good dyeablility with cationic dyes and disperse dyes. The optimum level of blending was studied for tenacity and thermal stability of melt blended fibers. The wash fastness properties of the dyed fibers were high. 
TABLE 7. Dispersed and basic dyes used.

\begin{tabular}{ccccc}
\hline No. & Brand name & C.I.Number & M. wt & Migration rate \\
\hline $\mathbf{1}$ & Forcon Navy Blue S-2GLI & C.I.Disperse Blue 79 & 639 & 2 \\
$\mathbf{2}$ & Forcon Blue SE-RI & C.I.Disperse Blue 183 & 473 & $4-2$ \\
$\mathbf{3}$ & Forcon Orange E-RL.I & C.I.Disperse Orange 25 & 323 & \\
$\mathbf{4}$ & Sandocryl Blue B-3G & C.I. Basic Blue 3 & & \\
$\mathbf{5}$ & Sandocryl Red BLH & C.I. Basic Red 22 & \\
\hline
\end{tabular}

II. Dyeing Polypropylene using superfacial carbon dioxide.

An alternative dyeing method for PP using supercritical carbon dioxide was developed[20]. as follows:-
GAIKER has studied SC-CO $\mathrm{CO}_{2}$ dyeing of PP with an acid dye. Firstly, solubility of the dye in $\mathrm{SC}-\mathrm{CO}_{2}$ and co-solvent was tried [21]. Ways for introduction of PP samples and dye powder and the supercritical dyeing process parameters were studied. After process optimization, $\mathrm{SC}-\mathrm{CO}_{2}$

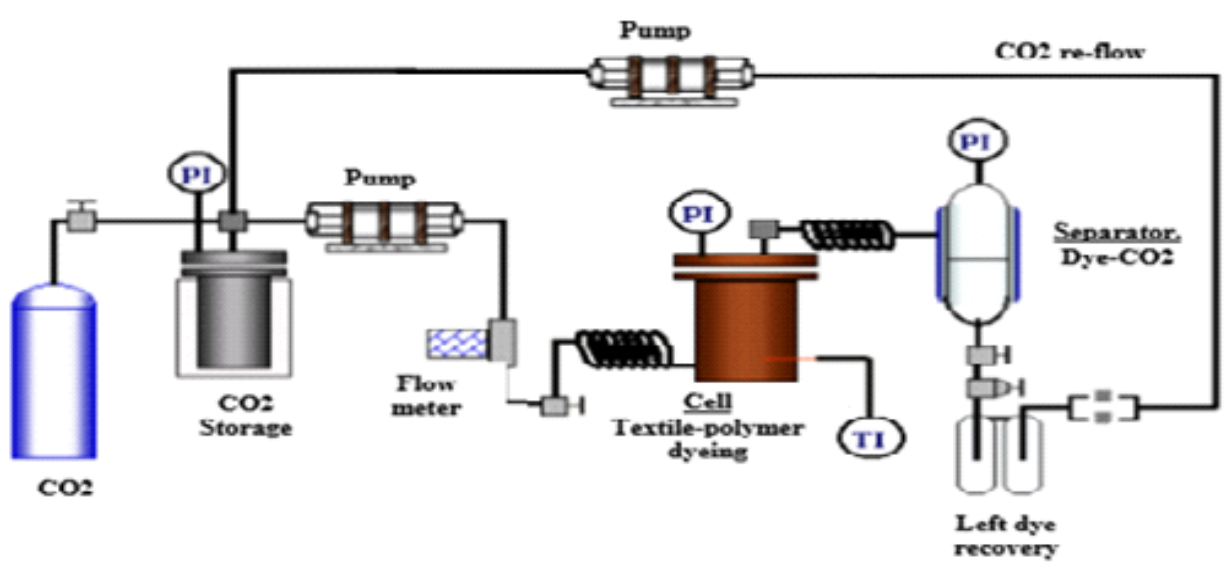

Fig. 7. One -step supercritical dyeing process.

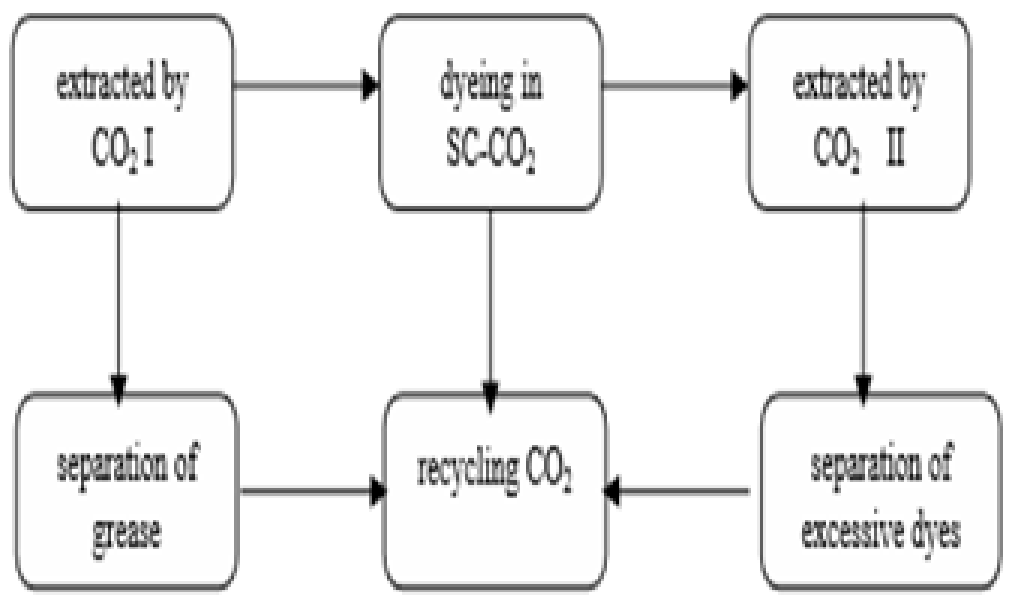

Fig. 8. Process of dyeing in SC-CO2. 


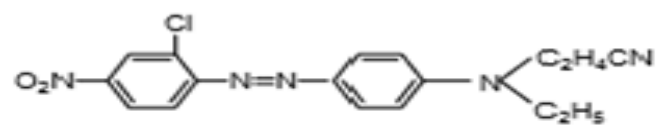

1.C.I. Disperse Red 50

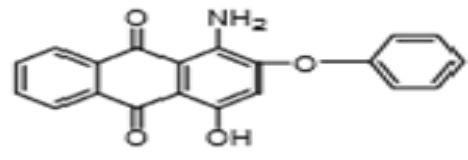

2. C. I. Disperse Red 60

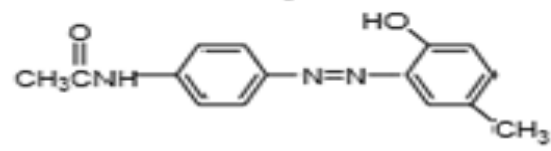

3. C.I. Disperse Yellow 3

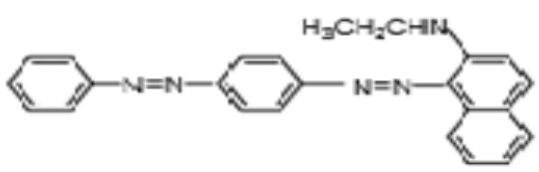

4. C.I.Solvent Red 19

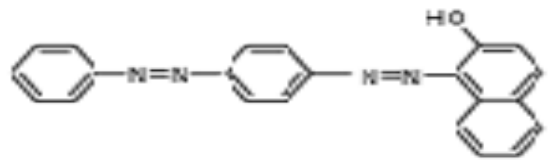

5. C.I. Solvent Red 23

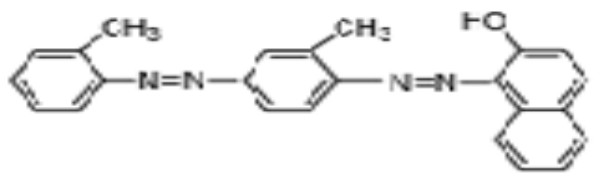

6. C.I.Solvent Rad 24

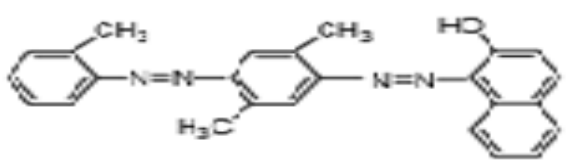

7. C.I. Solvent Red 26

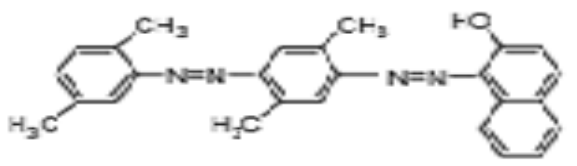

8. CI. Solvert 27

Fig. 9. Structures of disperse dyes used inSC-CO2.

dyeing of polymer was carried out at a pilot scale. Different PP characteristics were evaluated. Tests demonstrated that it is possible to dye PP fibers with this supercritical dyeing method and the dyed fabric was of the same quality as in aqueous bath. Polypropylene was dyed using $\mathrm{SC}-\mathrm{CO}_{2}$ [22] at pressure $10-33 \mathrm{MPa}$ and at $35-150{ }^{\circ} \mathrm{C}$ using special co-solvents to improve dye sorption.

The equilibrium sorption of dye increased with pressure, density, temperature and the optimum amount of co-solvent was studied. An indication is given to replace the current dyeing process with supercritical fluid dyeing process which does not give pollution problem and save energy.

\section{Mass Coloration with Pigments}

Colored PP fibers are prepared as spun dyed fibers. Mass coloration includes dispersion and homogenization of pigments or dyes in PP before extrusion and spinning [23]. Pigments are usually solid color particles insoluble in PP, also waterinsoluble. They are divided according to their origine into organic and inorganic. PP melt is, in principle, not colored by the dyes and pigments directly but by means of concentrated color dispersions called master batches.
Preparation of spun PP dyed fibers

To PP, dispersions are added in two methods:

- Weight dozers are added directly to the extruder feed;

- After melting and homogenization in a separate extruder the melt of the concentrate is dosed by injecting into the melt of PP. The melt mixture is then homogenized in a mixer. The content of the organic pigment usually varies from $0.01-1.5 \mathrm{wt}$ \% $\%$ in the colored melt, according to the required color strength needed.

Some pigments accelerate crystallization of PP stream below the spinneret, e.g. phthalocyanine pigments. Others, such as carbon black and $\mathrm{Ti}_{2}$, are less effective on the kinetics of crystallization.

\section{Mass coloration with metallic additives}

The influence of colored organic and inorganic pigments on the physical properties of molten PP such as CI yellow 37 and CI pigment Red 108 on tensile strength, abrasion resistance of mass coloration of PP has been investigated [24].

\section{Inorganic pigments}

The most important inorganic pigments are oxides, sulfides, selenides, and chromate 
<smiles>COCCCn1c(=O)c2cc3c(=O)c4ccccc4c(=O)c3cc2c1=O</smiles>

C. I. Disperse Blue 60<smiles>Nc1c(Oc2ccccc2)cc(O)c2c1C(=O)c1ccccc1C2=O</smiles>

C. I. disperse Red 60<smiles>CC(=O)OCCN(CCC#N)c1ccc(N=Nc2c(Cl)cc([N+](=O)[O-])cc2Cl)cc1</smiles>

C. I. Disperse Orange 30<smiles>O=C1c2ccccc2C(=O)C1c1nc2ccccc2cc1O</smiles>

C. I. Disperse Yellow 54

Fig. 10. Molecular structure of the used Disperse Dyes in SC-CO2 method.

compounds of metals, mainly $\mathrm{Zn}, \mathrm{Ti}, \mathrm{Cd}, \mathrm{Cr}$, Ni, $\mathrm{Co}, \mathrm{Pb}, \mathrm{Mo}$. Carbon black is also included into this group. They have high thermo stability and good dispersibility in PP.

\section{Dyeing PP by other methods}

PP was chlorinated by sodium hypochlorite and subsequent dyed with 12 azo disperse dyes of basic character in both alkaline and acidic medium. The affinity of the dyes for chlorinated PP was described [25].

Chemical structure of nanoclay produces dye site for nanocomposites [26]. Polypropylene/ nanofil, a natural montmorillonite modified with benzyl tallow dimethyl ammonium salt composites were prepared by melt mixing and pressing. at different weight ratios. Maleic anhydridemodified polypropylene (PP-g-MA) was used as a compatibilizer. SEM images show that clays were dispersed into PP matrix in the form of large and small non isometric aggregates. However, the size of aggregates in the sample with $2 \mathrm{wt} \%$ nanoclay and $4 \mathrm{wt} \%$ PP-g-MA was larger. Thermal properties were not affected but the percentage crystallinity and the mechanical properties were reduced slightly. Films could be dyed with a dispersed dye with acceptable fastness and the shade changes with changing the clay content.

PP fibre dyeable with natural dyes was done with the incorporation of hyperbranched polymer (HBP) via melt spinning technique [27]. Walnut shell oak gall, dyer's camomile, and cehri were selected. The effect of HBP concentration on K/S values of natural dyed PP fibers was evaluated. The wash fastness and Fourier Transform Infrared Spectroscopy of fiber samples were also studied. The percent crystallinity of modified fibers was determined by Differential Scanning Calorimeter and the morphological analyses were performed with Scanning Electron Microscopy. It was observed that the addition of HBP increased the

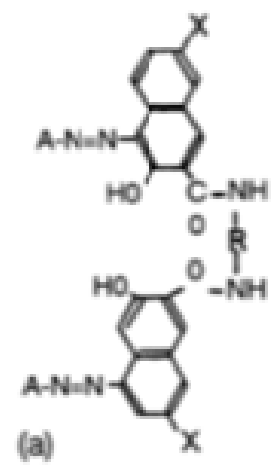

(b)

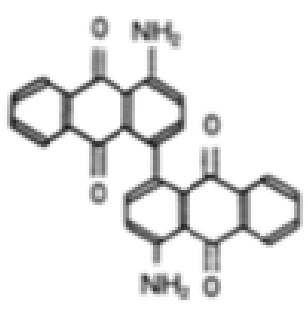

b)

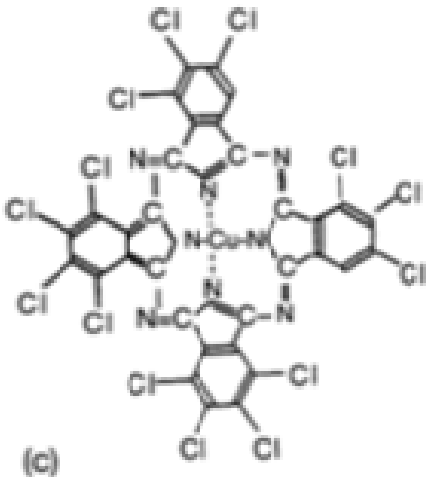

Fig.11. Pigments for spun dyed PP fibers: (a) C.l. Pigment Red 144; (b) C.I. Pigment Red 177; (c) C.I. Pigment Green 7.

Egypt. J. Chem. 62, No.1 (2019) 
dyeability of PP fiber using walnut shell, oak gall and cehri significantly compared to dyers camomile. The incorporation of HBP enhanced the dyeability of PP fibers with natural dyes.

The hydrophobicity of new dyes synthesized was analyzed numerically for unmodified pure PP fibers [28]. The octanol-water partition coefficient $(\log \mathrm{P})$, which is one of molecular descriptions for hydrophobicity of organic compounds was obtained by a semi-empirical method using Chem $3 \mathrm{D}$ software. The dyes of higher $\log \mathrm{P}$ was around 5 , the affinity of the dyes towards unmodified PP fibers was substantial. In comparison to the new
PP dyes, the conventional disperse dyes have log $P$ values lower than 5 that exhibited poor affinity.

A novel synthesis method was effected for the production of LCB- PP via in situ ozonation [29] during reactive extrusion process in the presence of co-agent such as trimethylol propane triacrylate (TMPTA). Ozonation of molten PP molecules produces high free radicals to induce PP chain scission reactions during extrusion. LCB structures in PP molecules are formed when TMTPA was added. The amount of LCB produced increases with increase TMPTA concentrations. Long-chain branching PP showed a significant
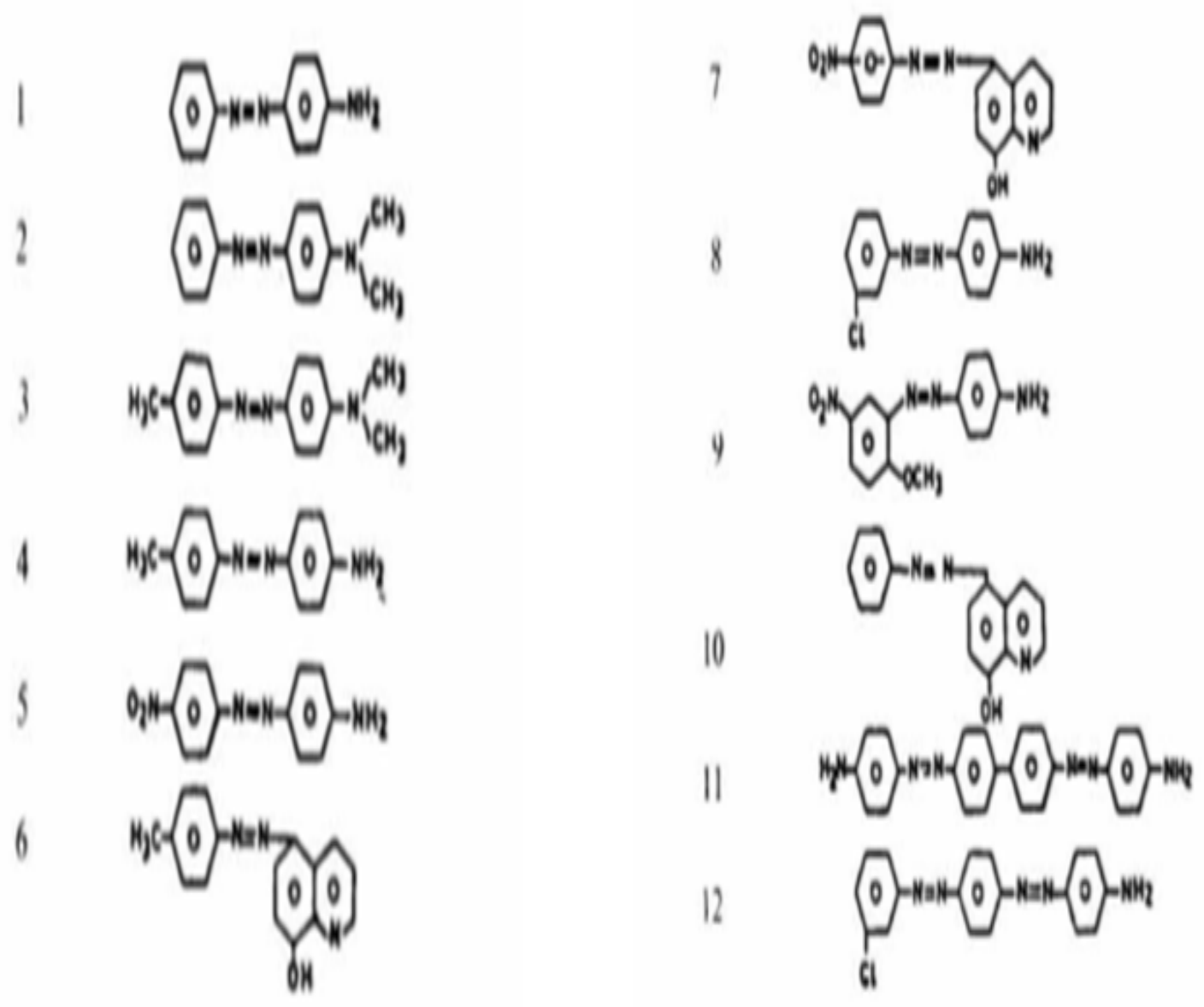

Fig. 12. Molecular structure of the 12 Azo Disperse Dyes used.

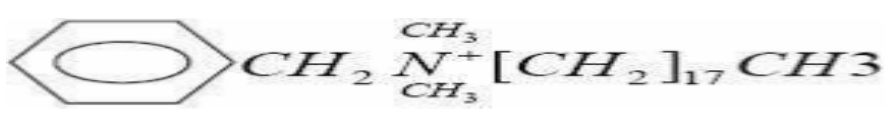

Fig. 13. Structure of benzyl tallow dimethyl ammonium salt. 
influence on the viscoelastic properties of the melts. The onset of the shear thinning region started at lower frequency and the Newtonian behavior disappeared gradually with the increase of TMPTA concentration. The continuous weight relaxation spectrum showed an additional longer relaxation time in LCB- PP spectrum, which indicated that the product was a mixture of linear molecules and branched molecules.

Dyeing properties of PP fabrics treated in dielectric barrier discharge (DBD) at atmospheric air pressure and padded with butane tetra carboxylic acid (BTCA) were evaluated [30]. Excess polar functional groups were created on the substrate surface after DBD pretreatment. BTCA was applied to PP fabrics by pad-dry-cure method.
Both treated and untreated fabrics were dyed using acidic and basic dyestuffs. The surface morphology was studied by Scanning Electron Microscopy. Treated samples were also characterized by means of Fourier Transform Infrared Spectroscopy. The wettability of the samples was evaluated by water drop test according to AATCC 79-2000 standard. Color intensities of the dyed fabrics were measured using a UV reflection spectrophotometer, in the range of 400-700 nm. For the treated PP fabric, the relative color strength (K/S values) of the dyed fabrics has been increased. The tensile strength test in both weft and warp directions, does not differ Also, the fastnesses properties of samples were done.
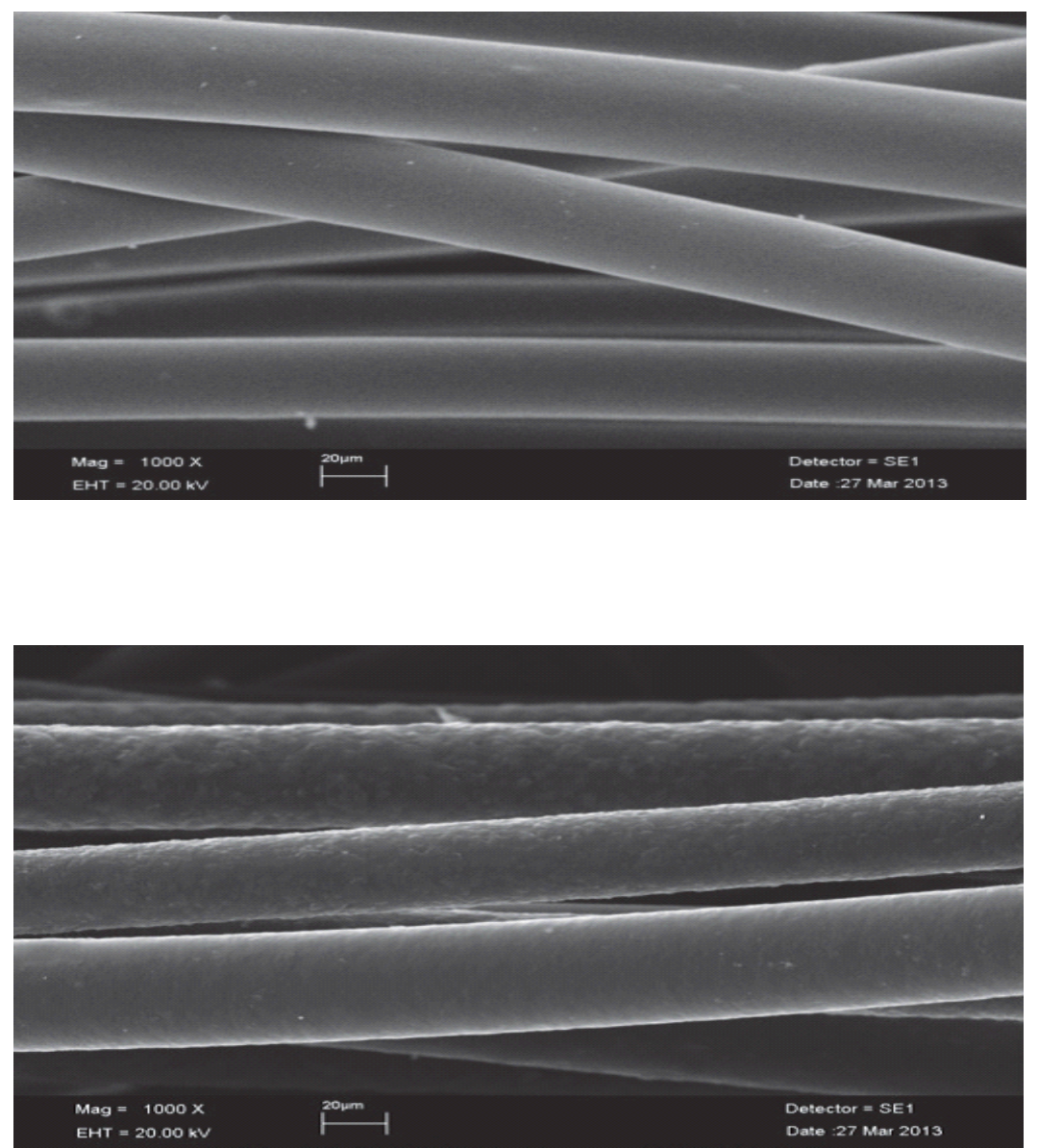

Fig.14. SEM images of fiber samples at magnification of PP (unmodified polypropylene) and MD-PP (3\% HBP modified polypropylene). 


\section{Conclusions}

Nonwoven PP fabrics are very important for the Textile industry. They have cheap price, wide applications in addition to possess high tensile strength mechanical properties and chemical resistance. $\mathrm{PP}$ has no reactive functional groups in their chemical structure and are difficult to be dyed by the conventional dyeing methods. PP fabrics are modified by different methods to improve their dyeing behavior by the following methods:

1. Blending PP chips with other polymers such as PTT, PET, PE, EVA, PS, PA etc...at different percentage ratios prior or during melt extrusion to improve dyeing.

2. Dyeing PP with disperse dyes in superficial carbon dioxide.

3. Mass coloration with organic, inorganic pigments or metallic additives in a melt extrusion device.

4. Dyeing PP by other methods.

5. PP modification by radio- grafting technique and dyeing [31-35].

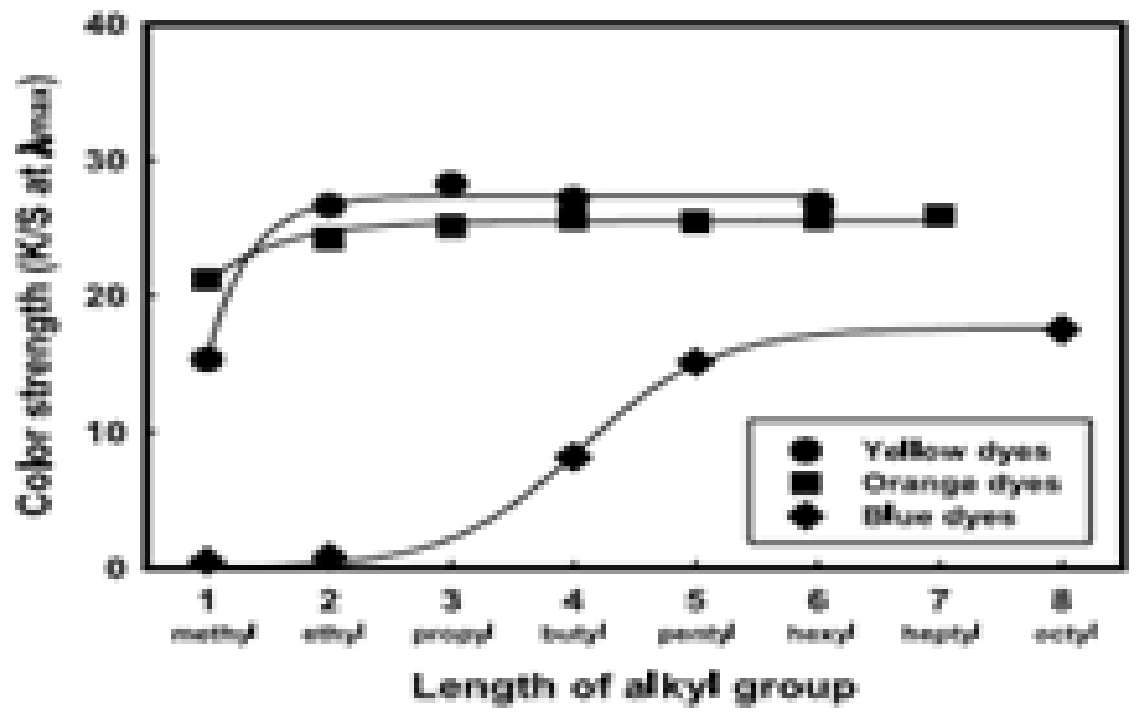

Fig. 15. Color strength of alkyl-substituted dyes onto PP fabrics at $130{ }^{\circ} \mathrm{C}$ for 1 hour. ( $\lambda$ max: Yellow dyes $420 \mathrm{~nm}$, Orange dyes 370nm, Blue dyes 600nm).

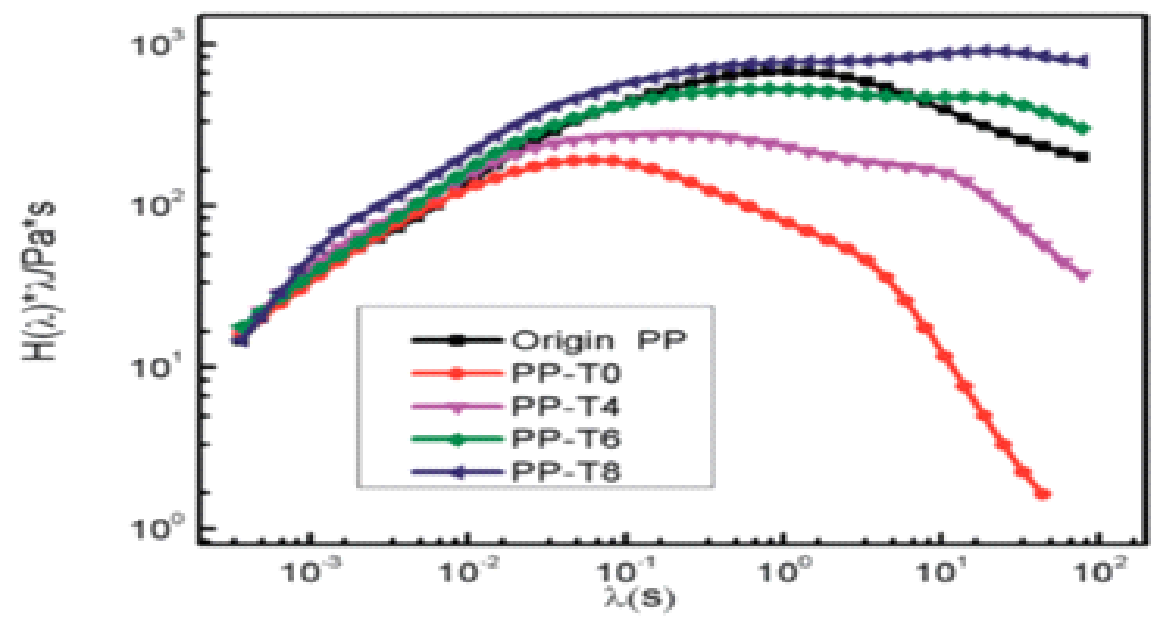

Fig. 16. Weighted relaxation spectrum of original PP and extruded PPs with different TMPTA concentrations (reaction temperature $210{ }^{\circ} \mathrm{C}$ polymer throughput $1 / 426.8 \mathrm{~g}$ min. 


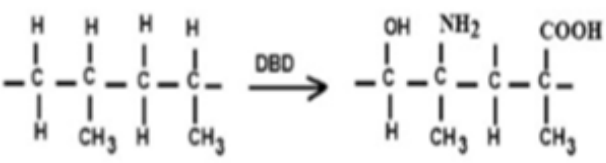

pp

DBD treated PP

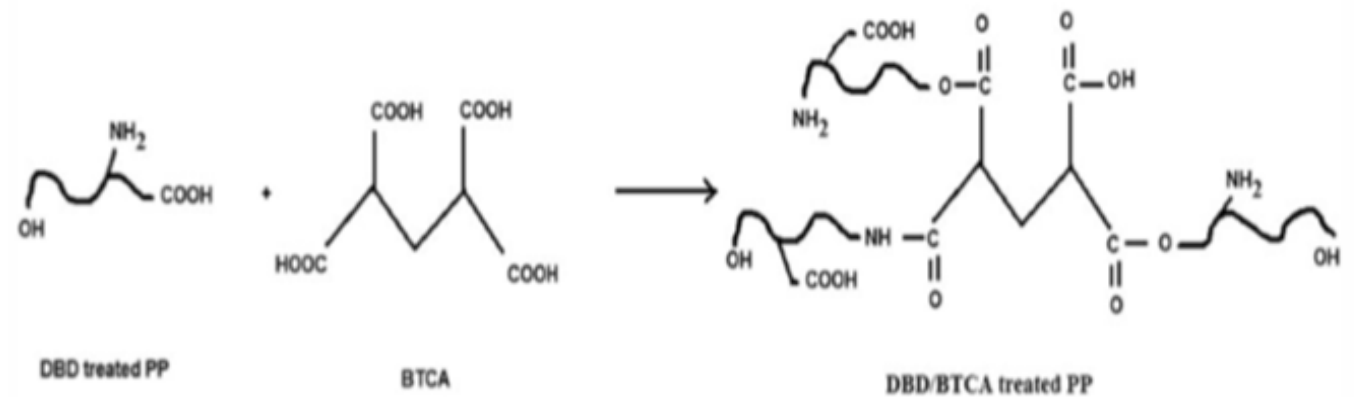

Fig. 17. Reaction mechanism between DBD-treated PP and BTCA.

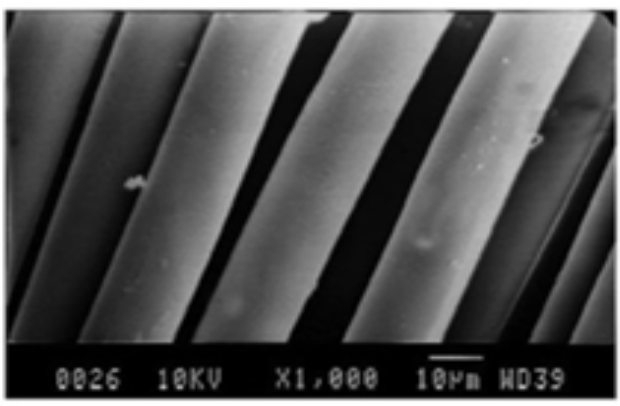

untreated PP fabric

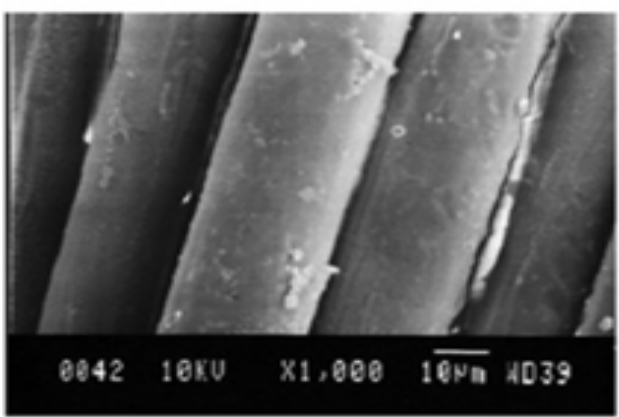

DBD treated PP fabric

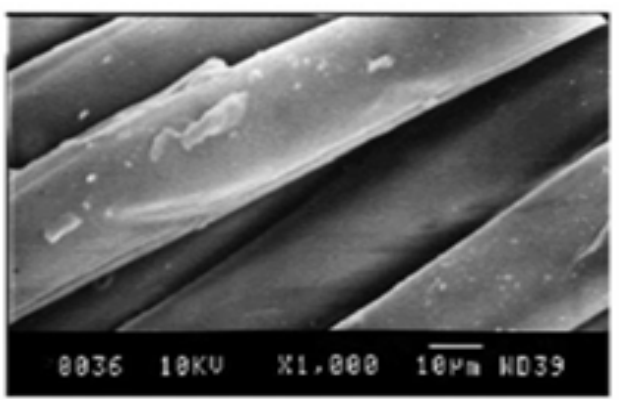

BTCA treated PP fabric

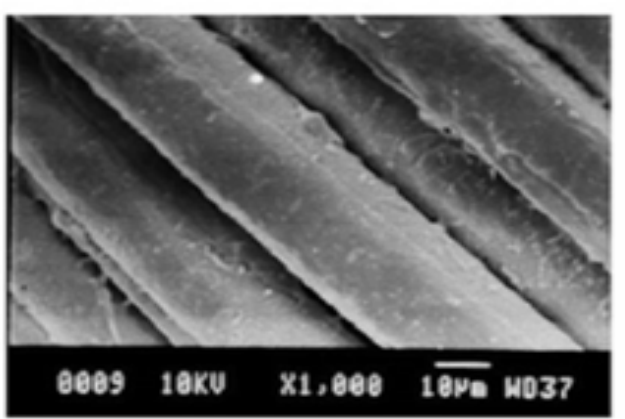

DBD/BTCA PP fabric

Fig. 18 SEM images of untreated and treated PP.

\section{References}

1. Teli M., Desail P., Polypropylene/poly (trimethylene terephthalate) melt blend fibres with enhanced dyeability, IJERT, 2 (7), 24-29 (2013).

2. Sahinbaskan B., Koçak E.D., Merdan N., Akalıne dyeing of polypropylene blends by using microwave energy, Journal of Engineered Fibers and Fabrics, 12 (2), 20-27 (2017).

Egypt. J. Chem. 62, No.1 (2019)
3. Yu C., Zhu M., Shong X, Che Y, Study on dyeability polypropylene fiber and its properties, J. Appl.Polym. Sci., 82, 3172-3176 (2001).

4. Kuo C.F. J., Lan W.L., Dong M.Y, Chen1 S.H., Lin F. S., Development of disperse dye polypropylene fiber and process parameter optimization Part I: development of dyeable polypropylene fiber and parameter optimization, Text. Research Journal; 
88 (1), 3-13 (2018).

5. Bolhová E., Ujhelyiová A., Val'ková K., Marcinčin A., Dyeing kinetics and colouristic properties of blend PP/PET fibres, Fibres \& Textiles in Eastern Europe; 5-6 (64), 131-135 (2007).

6. Mirjalili F., Moradian S., Amer F., Enhancing the dyeability of polypropylene fibers by melt blending with polyethylene terephthalate, The Scientific World Journal, Article ID 468542, 10 pages (2013).

7. Ujhelyiova A., Bolhova E., Oravkinova J., Tin`o R., Marcincin A., Kinetics of dyeing process of blend polypropylene/polyester fibers with disperse dye, Dyes and Pigments, 72 (2), 212-216 (2007).

8. Prchal V., Marcincin A., Kristofic M., Ondrejmiska K., Dyeing and colourist properties of polypropylene fibers, Fibers and Textiles, 1 (3), 136-138 (1994).

9. Marcincin A., Kostialova A., Kristofic M., Textile fibers from polymer blends, Fibers and Textiles, 5 (4), 210-212 (1998).

10. Burkinshaw S.M., Froehling P.E., Mignane M., The effect of hyper branched polymer on the dyeing of polypropylene fibers, Dyes and Pigments, 53 (3), 229-230 (2002).

11. Ujhelyiova A., Bolhova E., Crystallisation behaviour of polypropylene in blend PP/PET fibers, Fibers and Textiles, 10 (4), 163-167 (2003).

12. Ghasemi T., Tavanaie M.A., Safi M., Shushtari A. M., A study on dyeing behavior of modified PP fibers produced as poly blend fibers Part 1: The Effects of fibers draw ratio and disperse dyes molecular weight, JCST, 6 (2), 103 (2012).

13. Cegarra J., Puente P., Considerationon kinetics of the dyeing process of polyester fibers with dispersed dyes, Textile Research Journal, 37 (5), 343-350 (1967).

14. Marcinčin A., Ujheliyova A., Marcincinova T., Fiber - forming blends of polypropylene and polyethylene terephthalate, Macromolecular Symposia $17666-72$ (2001).

15. Seves A., Testa G., Marcandalli B., Bergamasco L., Munaretto G., Inducing water bath dyeability in polypropylenic fibers by their blending with polyamide 6, Dyes and Pigments; 35 (4), 367-373 (1997).

16. Kristofic M., Modified polypropylene fibers, Fibers and Textiles in Eastern Europe, 2, 38-39 (1994).

17. Ujhelyiova A., Bolhova E., Marcincin A., Tino R., Blended polypropylene/polyethylene terephthalate fibers: Crystallisation behavior of polypropylene and mechanical properties, Fires and Textiles, 154 (63), 26-29 (2007).

18. Son T. W., Lim S. K., Chang C. M., Kim S. S., Cho I. S., Physical modification of polypropylene: preparation of fibers dyeable with disperse dyes, Coloration Technology, 115 (2), 366-369 (1999).

19. Teli M. D., Adivarekar R. V., Ramani V. Y., Sabale A. G. , Imparting disperse and cationic dyeability to Polypropylene through melt blending, Fibers and Polymers 5 (4), 264-269 (2004).

20. Qiu Y Li., Fen Z.S., Liang H.E, Wei M.A, Zong Y. J., The dyeing of polypropylene fibers in supercritical fluid, The 19 Proceedings of the 3rd International Conference on Functional Molecules, China.

21. Gaiker, Polyamide and polypropylene textile dyeing using superfacial carbon dioxide (sc$\mathrm{CO}_{2}$ ), Melliand International (3), (1998).

22. Shim J.J., Dyeing of polyester, aramide and polypropylene fibers in supercritical $\mathrm{CO}_{2}$, The 6TH ISSF at Versaille, April 2-30 (2003).

23. Marcincin A., Dyeing of polypropylene fibers, PP 172-177, Springer link Publisher 3/3/2018.

24. Halbe A.V., Various appproaches for dyeing of polypropylene, http:www.fibre 2fashion.com/ industry-article/2415/various-approaches-fordyeing-of-polypropylen.

25. Samanta A.K., Sharma D.N., Dyeing of polypropylene with some azo disperse dyes of basic character via chlorination route, Indian Journal of Fibers and Textile Research, 20, 206210 (1995).

26. Bahrami S.H., Mirzaie Z., Polypropylene/modified nanoclay composite-processing and dyeability properties, World Applied Sciences Journal, 13 (3), 493-501 (2011).

27. Davulcu A., Investigation the dyeability of hyperbranched polymer modified polypropylene fibers with natural dyes, TEKSTIL ve KONFEKSIYON; 25 (1), 73-79 (2015).

28. Kim T., Jang K., Jeon S., Calculation and analysis of hydrophobicity of the dyes synthesized for unmodified polypropylene fibers using molecular 
descriptors, Journal of the Korean Society of Dyers and Finishers; 21 (5), 21-26 (2009).

29. He G.J., Yuan B..Yu, Zhang T.T., Zhu W.Li., In situ ozonolysis of polypropylene during extrusion to produce long-chain branches with the aid of TMPTA, RSC Adv. 22531-22539 (2017).

30. Shahidia S., Ghorannevissb M., Ilalic R., Karamic M., Miladic M., Dyeing properties of the atmospheric pressure plasma-treated polypropylene fabric subjected to butane tetra carboxylic acid, The Journal of The Textile Institute., 107(5), 636-644 (2016).

31. Gawish S. M., Gavet L., Chabert B., Electron beam irradiation of polypropylene fabric and grafting by acidic monomers, J. Am. Dyes. Rep., 78 (12), 54 (1989).

32. Gawish S. M., Gavet L., Chabert B., Electron beam irradiation of polypropylene fabric and grafting of acidic monomers, J. Am. Dyes. Rep., 79 (1) , 39 (1990).

33. Gawish S.M., Kantoush A., El -Naggar A.M., Mosleh S., Grafting of 2- N DMAE Methacrylate onto gamma irradiated polypropylene fabric, $J$. Appl. Polym. Sci.,44 (9), 1671-1677 (1992).

34. Gawish S.M., Kantoush A., El-Naggar A.M., Mosleh S., Gamma preirradiation and grafting of 2N-morpholino ethyl methacrylate onto polypropylene fabric, J. Appl. Polym.Sci., 57 (1), 45-53 (1995).

35. Gawish S. M., Quaternization of polypropylene grafted with $2 \mathrm{~N}$-morpholino ethyl methacrylate, $J$. Am. Dyes. Rep., 80 (12), 39 (1991).

(Received 9/9/2018; accepted 29/10/2018)
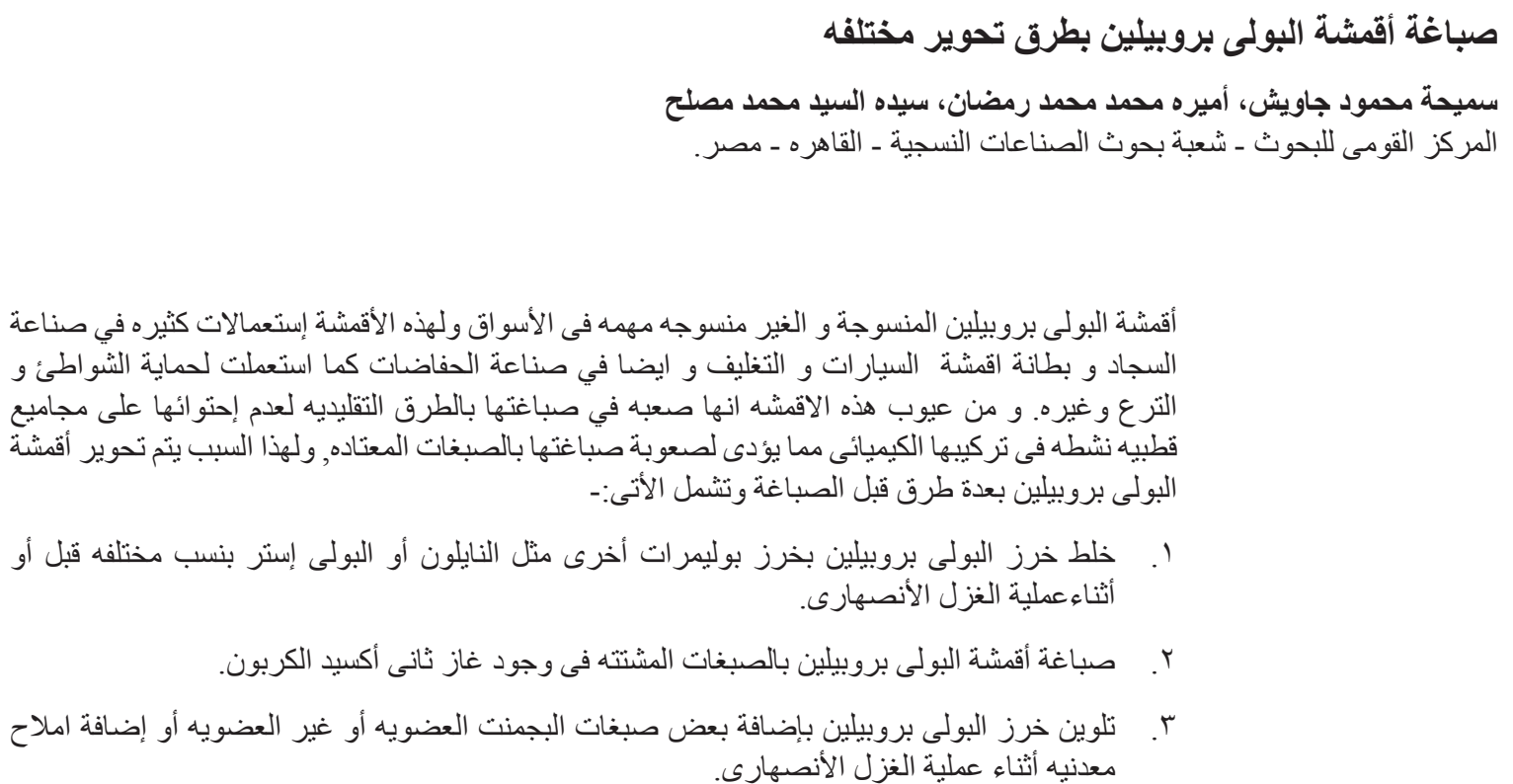